Review

\title{
Spermatogonial Stem Cells: A Current Update in the Management of Azoospermic Infertile Men
}

\author{
${ }^{1,3}$ Silvia W Lestari and ${ }^{2}$ Debby Aditya \\ ${ }^{I}$ Department of Medical Biology, Faculty of Medicine, Universitas Indonesia, Jakarta, Indonesia \\ ${ }^{2}$ Master Program in Biomedical Sciences, Faculty of Medicine, Universitas Indonesia, Jakarta, Indonesia \\ ${ }^{3}$ The Indonesian Reproductive Medicine Research and Training Center (INA-REPROMED)
}

Article history

Received: 26-09-2017

Revised: 23-11-2017

Accepted: 4-12-2017

Corresponding Author:

Silvia W Lestari

Department of Medical

Biology, Faculty of Medicine,

Universitas Indonesia, Jakarta, Indonesia

Email: finallysilvia@gmail.com

\begin{abstract}
One of the management of azoospermia is utilizing Assisted Reproductive Technologies (ART) by Intra-Cytoplasmic Sperm Injection (ICSI) with sperm obtained from the epididymis or directly from testis. In some conditions such as hypogonadism or undescended testis, the sperm are not successfully obtained. Thus, it is considered to perform Spermatogonial Stem Cells (SSCs) culture to restore men fertility. The objective of this work was to systematically review and discuss articles dealing with the subject of SSCs as a current update of azoospermic management. To this purpose, a computerized search of PubMed database was performed on the general term such as "male infertility", "azoospermia" and "human spermatogonial stem cells" and the articles obtained were evaluated and collected. SSCs are adult stem cells which capable to self-renewal, differentiate, transmit the material genetic to offspring. SSCs have a vital role in spermatogenesis and in vitro proliferation which could be utilized for azoospermic management. Some alternative of SSCs utilization followed by ICSI in men with azoospermia, the formation of artificial sperms and auto-transplantation of in vitro proliferated SSCs, are under the preclinical phase of development. SSCs utilization is the proper approach to manage the azoospermic infertile men who sperm couldn't be obtained. The procedure of SSCs utilization such as culture method, including isolation, identification and tumor forming, should be investigated prior to clinical application.
\end{abstract}

Keywords: Male Infertility, Azoospermia, Spermatogonial Stem Cells

\section{Introduction}

Infertility has become a major reproductive health and social concern afflicting 10 to $15 \%$ couples worldwide, in which male infertility contributes ranging from 2.5 to $12 \%$ of the cases (Wang et al., 2016; Cocuzza et al., 2013). Male infertility is considered by the manifestation of some conditions such as congenital urogenital abnormalities, malignancy, urogenital tract infection, temperature increment in the scrotum (varicocele), genetic defects of the endocrine system, genetic disorders and immunological factors (Cocuzza et al., 2013; Vlajkovic et al., 2012). Male infertility could be observed and diagnosed by a low count of sperm (oligozoospermia), decreased sperm motility (asthenozoospermia), abnormal morphology (teratozoospermia) and the absence of sperm (azoospermia). Azoospermia is defined as the absence of sperm in the ejaculate which identified in approximately
10 to $15 \%$ of infertile males (Cocuzza et al., 2013). One of the management of azoospermia is utilizing Assisted Reproductive Technologies (ART) by Intra-Cytoplasmic Sperm Injection (ICSI) which sperm retrieved from the epididymis by percutaneous/microsurgical epididymal sperm aspiration (PESA/MESA) or directly from testis by testicular sperm aspiration/extraction (TESA/TESE). In some conditions such as hypogonadism or undescended testis, the sperm are not successfully retrieved. Thus, it is considered to perform Spermatogonial Stem Cells (SSCs) to manage these infertile men (Goossens and Tournaye, 2014; Vassena et al., 2015).

\section{Spermatogonial Stem Cells as a Potential Management of Azoospermic Infertile Men}

SSCs are adult stem cells which reside in the basal layer of the seminiferous tubules, surrounded by Sertoli cells. SSCs capable to self-renewal, differentiate, 
transmit the material genetic to offspring. SSCs have a vital role in spermatogenesis by providing sperm production throughout life in men (Vassena et al., 2015; Yang et al., 2014). Recent studies have demonstrated that SSCs have a potential role in male infertility management (Wang et al., 2016). However, a rarity and limitation of the specific biomarker of SSCs could be one of significant challenge in SSCs utilization (Yeh et al., 2009). Recently, SSCs utilization followed by ICSI in infertile men, the formation of artificial sperms and autotransplantation of in vitro proliferated SSCs, are in under the preclinical phase of development. The optimum development of culture technique and auto-transplantation of SSCs would have enormous benefits, especially for the management of azoospermic infertile men.

One of the management of azoospermia is utilizing ARTs by ICSI which sperm obtained from the epididymis (PESA/MESA) or directly from testis (TESA/TESE), as long as spermatogenesis occurs. However, male infertile patients caused by hypogonadism or undescended testis, the sperm are not successfully obtained. Thus, it is considered to perform SSCs (Goossens and Tournaye, 2014; Sadri-Ardekani et al., 2014). Recently, there are some possibility of SSCs utilization followed by ICSI in male infertility management such as artificial sperms formation by culture and autotransplantation of in vitro proliferated SSCs which under preclinical phase of development.

\section{Culture Systems of In Vitro Germ Cell}

Several culture systems have been established in order to conduct optimal in vitro germ cell culture system which could maintain SSCs pluripotency or achieve sperm maturation as demonstrated in Table 1. The optimum condition of SSCs culture system is required in order to achieve in vitro spermatogenesis which might be used in ART. Three-dimension culture systems have been developed such as matrigel (Choi et al., 2014) and hydrogel (Guo et al., 2015) which could supports proliferation and long term maintenance of SSCs. Additionally, culture systems using coating plate (Koruji et al., 2012; Sadri-Ardekani et al., 2014; Goharbakhsh et al., 2013; Lim et al., 2010) and coculturing SSCs with somatic testicular tissue such as Sertoli cell (Zanganeh et al., 2013; Mohamadi et al., 2012; Rastegar et al., 2015; Liu et al., 2011; Akhondi et al., 2013) have been developed in order to maintain SSCs pluripotency. Elhija et al. (2011) studied about producing post meiotic germ cells including morphologically normal sperm which retrieved from testicular cells of immature mice in a soft agar culture system. Briefly, three research groups conducted the establishment of germ cells derived from mouse embryonic stem cells (Aponte et al., 2013). Geijsen et al. (2004) investigated in producing gamete-like cells from mouse embryo stem cells and using ICSI as a functional assay. Thus, embryo was obtained after fertilization and reached the blastocyst stage. The other research (Nayernia et al., 2006; Toyooka et al., 2003) studied in generating sperm in vitro, but only one of them using ICSI as a functional assay to obtain offspring. However, the low efficiency outcome was generated and the mice died prematurely (Nayernia et al., 2006). Recently, in vitro spermatogenesis from human SSCs has not been developed yet, even though in vitro spermatogenesis has been successfully conducted in rodents resulting in the healthy offspring after ICSI (Goossens and Tournaye, 2014).

The authors assessed that the isolation, culture, expansion, and identification technique of SSCs should be optimized in order to make SSCs feasible in research and clinical medicine. Some limitations still restrain the welldevelopment of SSCs culture system. As has known various factors could influence SSCs culture condition such as conditioned-medium, serum and feeder cells utilization, hormonal treatment. An effort in order to mimic the microenvironment of the testes to in vitro testicular model has been a challenge in growing sperm successfully.

Table 1: In-vitro germ cell culture system

\footnotetext{
Spermatogonial Stem Cells Culture

Development of three-dimension culture systems to support proliferation and long term maintenance of SSCs.

Development of coating plate culture systems which capable to support the proliferation and self-renewal of SSCs.

Co-culturing SSCs with somatic testicular tissue such as

Sertoli cell as feeder layer could support

proliferation and differentiation of SSCs.

Expansion and differentiation of testicular cells from 7-day-old immature mice in a soft agar culture system, thus post meiotic germ cells were produced including morphologically normal sperm. Producing gamete-like cells from mouse embryo stem cells and using ICSI as a functional assay. Embryo was obtained after fertilization and had reached the blastocyst stage.

Generating mouse sperm in vitro and using ICSI as a

functional assay to obtain offspring.

Generating sperm in vitro from mouse embryo stem cells.
}

Reference

Choi et al. (2014; Guo et al., 2015).

Koruji et al. (2012; Ardekani et al., 2009;

Goharbakhsh et al., 2013; Lim et al., 2010).

Zanganeh et al. (2013; Mohamadi et al., 2012;

Rastegar et al., 2015; Liu et al., 2011; Akhondi et al., 2013).

Elhija et al. (2011).

Geijsen et al. (2004).

Nayernia et al. (2006).

Toyooka et al. (2003). 
Nevertheless, obtaining sufficient SSCs clinical utilization is crucial, therefore establishment of a stable culture system which could maintain propagation of SSCs in vitro is required. In the future decade, authors believed that developing the stable culture system of SSCs will be an attainable goal, yet the time for the successful in vitro differentiation is less predictable.

\section{Spermatogonial Stem Cells Transplantation}

Several researches of SSCs transplantation have been conducted in which used as a functional assay and potential fertility men restoration possibility as shown in Table 2. SSCs transplantation is suggested could contribute to re-establish men fertility by increasing in vivo spermatogenesis possibility followed by natural conception achievement or ARTs by ICSI. Breakthrough of SSCs transplantation by Brinster and Zimmermann (1994) inspired the onset of testicular stem cell research in which became the cornerstone of germ line stem cell utilization. They investigated SSCs transplantation which derived from wild type mouse into the testes of chemotherapy-treated mice to restore spermatogenesis. In the recipient testes, the transplanted SSCs showed capability to expand and differentiate forming mature sperm and restore spermatogenesis. The result showed that donor SSCs could engraft in testes, re-establish spermatogenesis, thus leading to the offspring production. Human SSCs auto-transplantation has been conducted firstly by Radford et al. (1999) according to recent scientific literature. They introduced cryopreservation and auto-transplantation human SSCs from 12 male non-Hodgkin's lymphoma patients who underwent chemotherapy. Seven patients had the cells re-infused back into their testes but the outcome is remain unknown because there have been no follow-up reports on the fertility status. Recently, Choi et al. (2014) developed a three dimension of culture system for long term culture of mouse SSCs and SSCs functionality was confirmed by transplanting in vitro proliferated mouse SSCs to chemotherapy-treated mice. Whilst Sadri-Ardekani et al. (2009) and Koruji et al. (2012) transplanted human SSCs into the seminiferous tubules of chemotherapy -treated mice, hence the presence of transplanted cells was detected in the basal compartment of the seminiferous tubules in the recipient testes after xenotransplantation. The authors assessed according to published reports that transplanting in vitro proliferated SSCs into recipient could be used to receive functional sperm in which capable of producing fertile offspring. SSCs transplantation has several benefits such as allowing the possibility to auto-transplantation in human which could allow natural conception. Even though SSCs transplantation in mice and other higher mammals has been successful, several considerations still restrain the application of this technology in humans (Vassena et al., 2015; Valli et al., 2014).

Some limitations upward ahead are the limited number of SSCs which could be obtained is suggested not sufficient enough to recolonize and restore fertility, hence it should be propagated in vitro previously. Furthermore, auto-transplantation of SSCs is a remaining risk of tumor forming and invasive procedure requirement is needed. Additionally, the health quality of offspring including genetic and epigenetic alteration has not been extensively studied yet (Liu et al., 2011). Nevertheless, based on previously research, there is a chance and it should be possible to transplant testicular tissue or cells, as the potential management of azoospermic man, for regeneration of spermatogenesis in the future time.

\section{The Challenge of SSCs Utilization}

The authors addressed that there are some challenges up forward regarding to utilization of SSCs in clinical application as presented in Table 3. Like another adult stem cells, SSCs has a capability of unlimited proliferation, there is a possibility leading to tumor formation if its characteristic was altered (Kubota et al., 2006). Another consideration, transplantation of in vitro proliferated SSCs culture could be contaminated with tumor cells, as well as the un-growth cells, thus also driving to tumor formation, even there were several methods to identify the SSCs yet the possibilities still remained.

Table 2: The transplantation of spermatogonial stem cells

The Transplantation of Spermatogonial Stem Cells
Transplantation of mouse SSCs into the chemotherapy-treated mice.
Donor SSCs could engraft and re-establish spermatogenesis,
thus leading to the offspring production.
Introducing cryopreservation and human SSCs auto-transplantation
from 12 male non-Hodgkin's lymphoma patients who underwent
chemotherapy. Seven patients had the cells injected back into their
testes, yet the outcome is unknown.
Transplantation of mouse SSCs into the chemotherapy-treated mice.
SSCs was confirmed and found in the recipient testicular tubules.
Xenotransplantation of human SSCs into the seminiferous tubules of
chemotherapy -treated mice. The presence of transplanted cells was
detected in the basal compartment of the seminiferous
tubules in recipient testes.
Reference

Brinster and Zimmermann (1994; Avarbock et al., 1996).

Radford et al. (1999).

Choi et al. (2014).

Koruji et al. (2012; Sadri-Ardekani et al., 2009). 
Table 3: The challenge of spermatogonial stem cells utilization

\begin{tabular}{ll}
\hline The Challenge of Spermatogonial Stem Cells Utilization & Reference \\
\hline Defining a method and culture system & Gassei and Orwig (2016) \\
Possibility of epigenetic or genetic alteration & Samplaski et al. (2014) \\
Possibility of tumor formation & Kubota and Brinster (2006) \\
Should be increased in number of SSCs prior to utilization & Samplaski et al. (2014) \\
Functionality and mechanism of SSCs in regenerating spermatogenesis & Gassei and Orwig (2016) \\
Ethical concern & Kubota and Brinster (2006) \\
\hline
\end{tabular}

Generally the number of SSCs obtained usually in limited number, it should be propagate for obtaining an adequate amount of cells in advance of autotransplantation to the patient. Nevertheless, in vitro culture system still could not assure the integrity of genetic material of SSCs yet. There is little research conducted on any genetic and epigenetic alteration in either the transplanted SSCs or offspring (Samplaski et al., 2014). During cells propagation through culture, it is possible in occurring of epigenetic or genetic alteration although the offspring obtained seen to be healthy in animal models. Exposure of growth factor, serum and the maturation processes contribute to genetically modification. Particularly, male germ cells undergo epigenetic remodeling during spermatogenesis, errors during the process may cause altered genetic information to be transmitted to offspring generating in abnormalities. The health quality of offspring could be affected, as of the genetic and epigenetic screening of the cells is required in advance of human SSCs utilization in clinical application.

There is not gold standard in order to measure the functionality of human SSCs, even transplantation to restore spermatogenesis with functional sperm and offspring in animal model already conducted (Gassei and Orwig, 2016). Nowadays, the successful of generating offspring through SSCs transplantation could be observed in mice, rat and other mammals, but there is an ethical consideration of stem cell based therapy especially SSCs in human. Another interest point under investigation is the mechanism of SSCs in re-establish spermatogenesis, as well as another kind of stem cells, whether the injected cells could proliferate or the paracrine factor which suggested stimulate the reside stem cells is remain unknown. Despite many challenges ahead, SSCs utilization is one of promising agent which could be used as potential management of azoopermic men in the future.

\section{Conclusion}

In conclusion, after reviewing the recent scientific literature, to date, even though there are no stem cell based therapies which available, some of the clinical trials are being held to ameliorate or solve the reproductive medicine issues such as male infertility. SSCs utilization is the proper approach to manage the azoospermic infertile men whose sperm couldn't be retrieved. In vitro production of sperm and autologous transplantation are currently under clinical trial phase development. Surely, the procedure of SSCs utilization such as culture method including isolation, purification, identification, and functional assay as well as possible tumor formation should be investigated prior to clinical application.

\section{Acknowledgement}

The authors would like to express gratitude to the Hibah Penelitian Dasar Perguruan Tinggi (PDUPT) at Universitas Indonesia for supporting this review article.

\section{Authors' Contribution}

Silvia W Lestari: First author of the manuscript, journal collection and supervisory role of the review article.

Debby Aditya: Journal collection and manuscript review article.

\section{Ethics}

This review article is original and contains unpublished material. The corresponding author confirms that all of the authors have read and approved the manuscript and no ethical issues involved.

\section{References}

Akhondi, M.M., A. Mohazzab, M. Jeddi-Tehrani, M.R. Sadeghi and A. Eidi et al. Propagation of human germ stem cells in long-term culture. Iran J. Reproductive Medicine, 11: 551-8.

Aponte, P.M., S. Schlatt and L.R. de Franca, 2013. Biotechnological approaches to the treatment of aspermatogenic men. Clinics, 68: 157-67. DOI: 10.6061/clinics/2013(Sup01)18

Avarbock, M.R., C.J. Brinster and R.L. Brinster, 1996. Reconstitution of spermatogenesis from frozen spermatogonial stem cells. Nature Medicine, 2: 693-6.

Brinster, R. and J.W. Zimmermann, 1994. Spermatogenesis following male germ-cell transplantation. Proceedings of the National Academy of Sciences of the United States of America. 91: 11298-302.

Choi, N.Y., Y.S. Park, J.S. Ryu, H.J. Lee and M.J. Arauzo-Bravo et al., 2017. A novel feeder-free culture system for expansion of mouse spermatogonial stem cells. Molecules Cells, 37: 473-9.

DOI: $10.14348 /$ molcells.2014.0080 
Cocuzza, M., C. Alvarenga and R. Pagani, 2013. The epidemiology and etiology of azoospermia. Clinics, 68: 15-26. DOI: 10.6061/clinics/2013(Sup01)03

Elhija, M.A., E. Lunenfeld, S. Schlatt and M. Huleihel, 2011. Differentiation of murine male germ cells to spermatozoa in a soft agar culture system. Asian J. Andrology, 14: 285-93.

DOI: $10.1038 /$ aja.2011.112

Gassei, K. and K.E. Orwig, 2016. Experimental methods to preserve male fertility and treat male factor infertility. Fertility Sterility, 105: 256-66.

DOI: 10.1016/j.fertnstert.2015.12.020

Geijsen, N., M. Horoschak, K. Kim, J. Gribnau and K. Eggan, 2004. Daley GQ. Derivation of embryonic germ cells and male gametes from embryonic stem cells. Nature, 427: 148-54.

DOI: 10.1038 /nature02247

Goharbakhsh, L., A. Mohazzab, S. Salehkhou, M. Heidari and A.H. Zarnani et al., 2013. Isolation and culture of human spermatogonial stem cells derived from testis biopsy. Avicenna J. Medical Biotechnol., 5: 54-61.

Goossens, E. and H. Tournaye, 2014. Male fertility preservation, where are we in 2014? Ann Endocrinol., 75: 115-7.

DOI: 10.1016/j.ando.2014.03.011

Guo, Y., L. Liu, M. Sun, Y. Hai and Z. Li et al., 2015. Expansion and long-term culture of human spermatogonial stem cells via the activation of SMAD3 and AKT pathways. Experimental Biology Medicine, 240: 1112-22. DOI: $10.1177 / 1535370215590822$

Koruji, M., A. Shahverdi, A. Janan, A. Piryaei and M.R. Lakpour et al., 2012. Proliferation of small number of human spermatogonial stem cells obtained from azoospermic patients. J. Assisted Reproduction Genetics, 29: 957-67.

DOI: $10.1007 / \mathrm{s} 10815-012-9817-8$

Kubota, H. and R.L. Brinster, 2006. Technology insight: In vitro culture of spermatogonial stem cells and their potential therapeutic uses. Nature Clinical Practice Endocrinology Metabolism, 2: 99-108. DOI: $10.1038 /$ ncpendmet0098

Lim, J.J., S.Y. Sung, H, Kim, S.H. Song and J. Hong et al., 2010. Long-term proliferation and characterization of human spermatogonial stem cell obtaines from obstructive and non-obstructive azoospermia under exogenous feeder-free culture conditions. Cell Proliferation, 43: 405-17.

DOI: $10.1111 / \mathrm{j} .1365-2184.2010 .00691 . \mathrm{x}$

Liu, S., Z. Tang, T. Xiong and W. Tang, 2011. Isolation and characterization of human spermatogonial stem cells. Reproductive Biology Endocrinology.

DOI: $10.1186 / 1477-7827-9-141$
Mohamadi, S.M., M. Movahedin, S.M. Koruji and M.A. Jafarabadi et al., 2012. Comparison of colony formation in adult mouse spermatogonial stem cells developed in Sertoli and STO coculture systems. Andrologia, 44: 431-7.

DOI: $10.1111 / \mathrm{j} .1439-0272.2011 .01201 . \mathrm{x}$

Nayernia, K., J. Nolte, H.W. Michelmann, J.H. Lee and K. Rathsack et al., 2006. In vitro-differentiated embryonic stem cells give rise to male gametes that can generate offspring mice. Developmental Cell, 11: 125-32. DOI: 10.1016/j.devcel.2006.05.010

Radford, J., 2003. Restoration of fertility after treatment for cancer. Hormone Res., 59: 21-3.

DOI: $10.1159 / 000067840$

Radford, J., S. Shalet and B. Lieberman, 1999. Fertility after treatment for cancer. Questions remain over ways of preserving ovarian and testicular tissue. British Medical J., 319: 935-6. DOI: $10.1136 / \mathrm{bmj} .319 .7215 .935$

Rastegar, T., M.H. Roudkenar, S. Parvari and M. Baazm, 2015. The interaction between sertoli cells and leukemia inhibitory factor on the propagation and differentiation of spermatogonial stem cells in vitro. Iranian J. Reproductive Medicine, 13: 679-86.

Sadri-Ardekani, H. and A. Atala, 2014. Testicular tissue cryopreservation and spermatogonial stem cell transplantation restore fertility: From bench to bedside. Stem Cell Research Therapy.

DOI: $10.1186 /$ scrt457

Sadri-Ardekani, H., S.C. Mizrak, S.K.M. van Daalen, C.M. Korver and H.L. Roepers-Gajadien et al., 2009. Propagation of human spermatogonial stem cells in vitro. J. Am. Medical Association, 302: 2127-34. DOI: 10.1001/jama.2009.1689

Samplaski, M.K., M. Deault-Bonin and K.C. Lo, 2014. Genetic and epigenetic changes after spermatogonial stem cell culture and transplantation. J. Int. Federation Clinical Chemistry Laboratory Medicine, 25: 027-41.

Toyooka, Y., N. Tsunekawa, R. Akasu and T. Noce, 2003. Embryonic stem cells can form germ cells in vitro. Proceedings National Academy Sci., 100: 11457-62. DOI: $10.1073 /$ pnas. 1932826100

Valli, H., B.T. Phillips, G. Shetty, J.A. Byrne and A.T. Clark et al., 2014. Germline stem cells: toward the regeneration of spermatogenesis. Fertil Steril., 101: 3-13. DOI: 10.1016/j.fertnstert.2013.10.052

Vassena, R., C. Eguizabal, B. Heindryckx, K. Sermon and C. Simon et al., 2015. Stem cells in reproductive medicine: ready for the patient? Human Reproduction, 30: 2014-21. DOI: $10.1093 /$ humrep/dev181

Vlajkovic, S., R. Cukuranovic, M.D. Bjelakovic and V. Stefanovic, 2012. Possible therapeutic use of spermatogonial stem cells in the treatment of male infertility: A brief overview. Scientific World J. DOI: $10.1100 / 2012 / 374151$ 
Wang, H., L. Wen, Q. Yuan, M. Sun and M. Niu, 2016. Establishment and applications of male germ cell and Sertoli cell lines. Reproduction. China. DOI: 10.1530/REP-15-0546

Yang, Q.E. and J.M. Oatley, 2014. Spermatogonial stem cell functions in physiological and pathological conditions. Current Topics Developmental Biology, 107: 235-67.

DOI: 10.1016/B978-0-12-416022-4.00009-3

Yeh, J.R. and M.C. Nagano, 2009. Spermatogonial stem cell biomarkers: improved outcomes of spermatogonial transplantation in male fertility restoration? Expert Review Molecular Diagnostic, 9: 109-14. DOI: 10.1586/14737159.9.2.109
Zanganeh, B.M., T. Rastegar, M.H. Roudkenar, I.R. Kashani and F. Amidi et al., 2013. Co-culture of spermatogonial stem cells with sertoli cells in the presence of testosterone and FSH improved differentiation via up-regulation of post meiotic genes. Acta Medica Iranica. 